\title{
Empirical evaluation of the ability to learn a calorie counting system and estimate portion size and food intake
}

\author{
Corby K. Martin*, Stephen D. Anton, Emily York-Crowe, Leonie K. Heilbronn, Claudia VanSkiver, \\ Leanne M. Redman, Frank L. Greenway, Eric Ravussin and Donald A. Williamson for the Pennington \\ CALERIE Team
}

Pennington Biomedical Research Center, 6400 Perkins Rd, Baton Rouge, LA 70808, USA

(Received 27 July 2006 - Revised 17 January 2007 - Accepted 9 February 2007)

\begin{abstract}
The aim of this study was to determine if: (1) participants could learn the HMR Calorie System ${ }^{\odot}$ by testing if their use of the system was more accurate after training; and (2) estimated portion size and food intake improved with training. A secondary aim was to use PACE (photographic assessment of calorie estimation) to assess if participants learned the HMR system. The PACE consists of pictures of foods, the energy content of which is known. A within-subjects design was used to test the aims of this study. Participants were 44 overweight $(25 \leq$ BMI $<30)$ adults who were randomly assigned to one of three calorie restriction groups or a weight maintenance group for 6 months. Participants attended weekly sessions and were trained to use the HMR system from weeks 5 to 8 . Participants were provided with foods to test if they could effectively use the HMR system and accurately estimate portion size and the amount of food eaten. The PACE was also used to quantify accuracy at using the HMR system. Training resulted in more accurate estimation of food intake, use of the HMR system and estimated portion size when presented with food. Additionally, training resulted in significantly more accurate use of the HMR system when measured with PACE. It is concluded that people can learn the HMR Calorie System ${ }^{\odot}$ and improve the accuracy of portion size and food intake estimates. The PACE is a useful assessment tool to test if participants learn a calorie counting system.
\end{abstract}

Portion size: Food intake: Calorie estimation: PACE: CALERIE

The prevalence of overweight and obesity has increased over the past 20 years (Flegal, 2005), and obesity is associated with a number of co-morbidities (Must et al. 1999). Although weight loss treatments promote modest short-term weight loss, weight regain is common (Perri \& Corsica, 2002). Inaccurate estimates of the energy content of food and food intake are likely reasons why many people fail to achieve a negative energy balance and lose weight, or maintain weight loss through energy balance (Hill, 2001).

A number of methods have been developed to help people estimate portion size more accurately, which should result in more accurate estimates of food intake; however, these methods are limited in their applicability and effectiveness. For instance, people can be trained to estimate the portion size of foods more accurately through the use of feedback, although this training does not necessarily generalise to foods that were not included in training (Rapp et al. 1998). A number of portion size measurement aids, including twoand three-dimensional models, have been developed, and their use improves portion size estimation, yet participants continue to under-report food intake (Howat et al. 1994). Moreover, use of these aids results in only modest improvements in accuracy, particularly when the differences in the size of food portions are small, which reflects many 'real world' situations (Brown \& Oler, 2000). An additional problem when using portion size measurement aids is large variability that negatively affects the accuracy of estimated food intake (Robson \& Livingstone, 2000). Importantly, underreporting of food intake is due to both underestimating the energy content of foods and failing to report all foods that were eaten. The focus of this paper is on improving the accuracy of the estimated energy content of foods and portion size.

The aforementioned literature indicates that methods are needed to train people to estimate food intake accurately and to quantify the accuracy of these estimates. Such methods should assist individuals in losing and maintaining weight loss since they could more accurately estimate food intake and adhere to calorie prescriptions. Moreover, a method to assess the accuracy of food intake estimates objectively would help clinicians identify people who inaccurately estimate food intake and who require additional resources and services. Indeed, Livingstone \& Robson (2000) note that methods for identifying misreporters are needed, and Cypel et al. (1997) report that researchers should improve the reporting of portion size measurement aids in the literature.

Abbreviations: CALERIE, Comprehensive Assessment of Long-term Effects of Reducing Intake of Energy; CR, calorie restricted; CR + EX, CR plus structured exercise; LCD, low-calorie diet; PACE, photographic assessment of calorie estimation.

* Corresponding author: Dr Corby K. Martin, fax +1 225-763-3045, email martinck@pbrc.edu 
The Health Management Resources Calorie System ${ }^{(0)}$ $\left(\mathrm{HMR}^{\mathrm{TM}}\right.$, Boston, MA, USA) is a promising approach for teaching people how to estimate food intake accurately. The HMR Calorie System ${ }^{\circledR}$ relies on anchor points or reference foods for which the energy content per serving is memorised. The HMR Calorie System ${ }^{\odot}$ has been used in very-low-calorie diets that include intensive lifestyle education. These programmes report good weight loss maintenance over 2 years (Anderson et al. 1992), but, to our knowledge, no study has empirically determined if people can learn to use the HMR Calorie System ${ }^{\odot}$ and if use of this system results in more accurate estimates of portion size and food intake $(\mathrm{kJ})$.

The PACE (photographic assessment of calorie estimation) was developed by the authors of this paper as a portable assessment tool. The PACE consists of pictures of foods, the portion size and energy content of which are known. The PACE can be used to quantify the accuracy with which people use a calorie counting system and it can provide people with practice using a calorie counting system. Because the portion size and energy content of the foods in the pictures are known, PACE could theoretically be used to quantify the accuracy of estimated portion size and the energy content of the foods represented in pictures. This is the first study to use PACE.

The primary aim of this study was to determine if participants could learn the HMR Calorie System $\left.{ }^{(}\right)$by testing if their use of the system's anchor points was more accurate after training and to determine if estimated portion size and food intake improved with training. A secondary aim was to use PACE to assess participants' accuracy at using the HMR system. A within-subjects design was used, where participants served as their own control. It was hypothesised that after training participants would be more accurate at using the HMR Calorie System ${ }^{\circledR}$, estimating portion size and estimating food intake when provided with foods. To our knowledge, this is one of the first studies to determine empirically if people can learn a calorie counting system and if training results in more accurate estimates of food intake.

\section{Methods}

\section{Participants}

Forty-four of 48 overweight $(25 \leq \mathrm{BMI}<30)$ adult $(25$ years $<$ age $<45$ years for females; 25 years $<$ age $<50$ years for males) participants enrolled in phase I of a multisite calorie restriction study entitled Comprehensive Assessment of Long-term Effects of Reducing Intake of Energy (CALERIE). Two participants withdrew from the CALERIE study and data collection for this paper began after two other participants had already begun the intervention; therefore, their data were not available for this report. Data reported herein were collected at the Pennington Biomedical Research Center, and a description of this centre's CALERIE phase I trial is reported elsewhere (Heilbronn et al. 2006). All participants provided written informed consent and the study was approved by the Institutional Review Board of the Pennington Center.

The data reported herein were collected using a within-subjects design, and participants served as their own control. Participants' accuracy in using the HMR system and estimating portion sizes and food intake was evaluated during a baseline phase before they were exposed to training, and after training. Hence, there was no control group that did not receive HMR system training, and this limitation is outlined in the Discussion. As part of the CALERIE trial, twelve participants were randomly assigned to each of four groups: (1) weight maintenance diet; (2) calorie restriction (CR; $25 \%$ restriction from baseline energy requirements); (3) CR plus structured exercise $(\mathrm{CR}+\mathrm{EX} ; 12.5 \% \mathrm{CR}$ plus $12.5 \%$ increase in energy expenditure via structured exercise); and (4) low-calorie diet (LCD; $3726 \mathrm{~kJ} / \mathrm{d}(890 \mathrm{kcal} / \mathrm{d})$ liquid formula diet until $15 \%$ of body weight is lost, followed by weight maintenance). The CALERIE study was 6 months in duration.

\section{Materials}

HMR Calorie System ${ }^{\circledR}$. The HMR Calorie System ${ }^{\odot}$ is a method to train people to estimate food intake accurately. The system consists of seven pages, and each page represents a food group, e.g. vegetables. On each page, a scale is presented that contains anchor points or reference foods for which the energy content per serving (cups, tablespoons or ounces) is memorised. The scale consists of a horizontal line, with lower energy foods on the left of the scale and higher energy foods on the right of the scale. Each page or scale has five to seven anchor points that are memorised. For example, broccoli is an anchor point and is $167 \mathrm{~kJ} / \mathrm{cup}$ (40 kcal/cup). When using the system, individuals determine if a food is an anchor point and, if so, they estimate the portion size of the food and multiply the portion size by the anchor point. If the food is not an anchor point, participants determine to what anchor point the food is most similar, using guidelines that are taught during the training. For example, a pear is not an anchor point, but it is similar to an apple, which is an anchor point. Therefore, the energy content of the pear is estimated by using the anchor point for an apple.

A pilot study was conducted to test if the HMR system's anchor points were accurate. The HMR system was used to determine the energy content ( $\mathrm{kJ}$ or $\mathrm{kcal}$ ) of 103 foods prepared by the Pennington Center's Metabolic Kitchen. The energy content of these foods was also calculated using Moore's Extended Nutrient (MENu) Database (2000), which is based on the USDA nutrient database (USDA, 2000). Correlation analysis and the Bland and Altman technique were used to test agreement between the HMR system and the $\mathrm{MENu}$ database. The correlation between energy estimates produced by the HMR System and the MENu database was very high $(r$ 0.97; $P<0.0001)$. Bland and Altman analysis indicated that on average the HMR system underestimated the energy content (kJ or kcal) of foods by only $21 \mathrm{~kJ}$ (standard deviation (SD) 72) $(5 \cdot 1$ (SD 17.1) kcal), yet this difference was significantly different from zero, $t(102) 3 \cdot 0, P<0 \cdot 05$. The Bland and Altman analysis indicated that this bias was consistent across different energy levels of foods, $F(102) 0 \cdot 78$, $P=0 \cdot 38$. Indeed, the $R^{2}$ from this analysis was very small (0.008). These findings indicate that the energy values produced by the HMR system provide a relatively accurate estimate of the energy content of foods compared with a validated nutrient database.

The HMR system was delivered to CALERIE participants as part of a comprehensive intervention that consisted 
of weekly group meetings where participants learned cognitive-behavioural strategies to adhere to calorie and exercise prescriptions. During the first 4 weeks of the intervention, participants were not exposed to the HMR Calorie System ${ }^{(0)}$, but they received formal training in use of the system from weeks 5 to 8 , and the system was reviewed during the group meetings for the rest of the trial.

Meal provision. Participants were provided with food from the Pennington Center's Metabolic Kitchen from weeks 1 to 12 and 22 to 24 . The portion size of the foods in these meals was carefully weighed and the energy content was calculated using the MENu database (2000) and ProNutra (version 3.0, Viocare, Princeton, NJ, USA). A $6 \mathrm{~d}$ menu cycle was developed and each of the six menus included breakfast, lunch and dinner. The meals were developed from a combination of $>100$ different foods, including meats, vegetables, soups, beverages, condiments, grains, fruits, breads and pastas. These were commonly consumed foods typical of a Western diet. For example, the pasta included spaghetti with red sauce, and the breads included white or wholemeal rolls and toast. Participants were primarily tested during dinner meals; therefore, they were exposed to no less than thirtyfour different foods. Each participant was provided with a specific calorie level determined individually and based upon group assignment. Participants in the LCD group did not receive meals until they achieved $15 \%$ reduction in body weight, which occurred, on average, by weeks 8 and 11 for males and females, respectively.

Data for three dependent variables were collected each week that food was provided to participants. Specifically, participants were asked to provide the following for each food in their meal: (1) the HMR anchor point ( $\mathrm{kJ}$ or kcal per unit of food); (2) estimated portion size; and (3) estimated food intake $(\mathrm{kJ})$. Data collection occurred immediately before the weekly group meetings, unless a participant was late to the group meeting. In these cases, data collection occurred immediately after the group meeting. During these evaluations, participants were not provided with feedback on their use of the HMR system or their accuracy at estimating portion size and food intake (participants were trained on the HMR system only in the group sessions). Additionally, although participants knew their daily calorie target, they did not know how much energy $(\mathrm{kJ})$ was in the test meal. Participants consumed all food provided; therefore, food intake was synonymous with the estimated energy content of the foods. Participants had no exposure to the HMR system's anchor points prior to week 5; therefore, they were asked to indicate the number of kcal per unit of measurement for each food, and the unit of measurement (cups, tablespoons, ounces) was provided to them only during this baseline assessment. Providing the correct unit of measure during baseline allowed us to compare participants' estimates from the baseline period with their estimates after training when they spontaneously used the correct unit of measure. Such comparisons necessitate that the units of measure are the same, since, for example, we are comparing tablespoons with tablespoons. If participants stated the incorrect unit of measure after the baseline assessment period, an accuracy score for the anchor point was not calculated, but an accuracy score for the portion size and food intake was calculated. Each dependent variable represented the participant's degree of accuracy, calculated as: accuracy $=(($ participant's estimate - actual $) /$ actual $) \times 100$. Therefore, a score of zero indicates perfect accuracy, 100 equals overestimation by $100 \%,-20$ equals underestimation by $20 \%$, etc.

PACE (photographic assessment of calorie estimation). The PACE method was developed based, in part, on the digital photography of foods methodology (Williamson et al. 2003, 2004), which demonstrates that people can be trained to estimate accurately the portion size and energy content of foods represented in pictures. In developing and validating the digital photography method, digital photographs were taken of six meals consisting of forty-five different foods, including meats, vegetables, soups, beverages, condiments, fruits, breads and pastas. Approximately ten photographs of each meal were taken to represent different portion sizes of the foods included in the meal. An additional forty photographs of breakfast, lunch and dinner foods were included. The portion size and energy content of the foods were measured and recorded by registered dietitians. These photographs were printed on $8.5^{\prime \prime}$ by $11^{\prime \prime}$ photographic paper. The anchor point, portion size and energy content of each of the foods were printed on the back of each picture. This system was named PACE. It was developed to quantify the accuracy of participants' use of calorie counting systems. Theoretically, PACE could also be used to quantify participants' accuracy at estimating the portion size and the energy content of the foods represented in the pictures.

The PACE was used to provide participants with practice using the HMR system and to quantify their accuracy in using the system weekly. Additionally, the participants' ability to estimate accurately the portion size and energy content of the foods represented in the pictures was quantified weekly. These methods were identical to the methods used when participants were presented with food, and accuracy scores were calculated using the same formula: accuracy $=($ (participant's estimate - actual)/actual) $\times 100$.

\section{Data analytic plan}

Accuracy scores for use of the HMR system, portion size and food intake were calculated weekly when participants were presented with food. The same accuracy scores were generated weekly when participants were presented with the PACE. The accuracy scores were averaged over 4-week periods, with weeks 1-4 serving as baseline. Missing value analyses were conducted to test if the missing completely at random assumption was met and to determine how missing values should be imputed.

To determine if training resulted in more accurate use of the HMR system and more accurate estimates of portion size and food intake, change scores were calculated to represent change in accuracy from baseline to the end of the trial (week 22-24 minus week 1-4). These change scores were calculated from the data collected when participants were provided with food and presented with the PACE. Analysis of covariance was used to test if the change scores differed by group (weight maintenance, CR, CR + EX, LCD). Baseline values were entered as covariates. $t$ tests were used to determine if the change scores differed from zero, thus indicating significant change or improvement. 


\section{Results}

\section{Participant characteristics}

A complete description of the study sample and weight loss data are reported elsewhere (Heilbronn et al. 2006). The majority of the sample identified themselves as white ( $n 30$, $63 \%$ ), followed by African-American ( $n$ 6, 33\%), and Asian or Latino ( $n 2,4 \%)$. The mean (SD) descriptive characteristics of the sample were: age 38 (SD 6.3) years, height 171 (SD 8.6) $\mathrm{cm}$, weight 81 (SD 10.5) $\mathrm{kg}$ and BMI 27.5 (SD 1.6) $\mathrm{kg} / \mathrm{m}^{2}$. The number of participants in each group who had data available for this study was: weight maintenance $=10, \quad \mathrm{CR}=12$, $\mathrm{CR}+\mathrm{EX}=11$ and $\mathrm{LCD}=11$.

Change in accuracy, measured with provided foods, associated with training

Missing value analysis was conducted for the accuracy data collected with provided food. Only one of thirty-three participants $(3 \%)$ was missing HMR, portion size and estimated food intake data at baseline. Expectation maximisation was used to impute this participant's values. Six of forty-four participants $(13.6 \%)$ were missing accuracy data at weeks 22-24. These data were imputed with expectation maximisaion because Little's chi-square test was not significant, $\chi^{2}(24) 34 \cdot 2, P=0 \cdot 08$, indicating that the missing completely at random assumption was met (Little \& Rubin, 1987). Expectation maximisation was used to replace missing values since it results in less distorted estimates compared with pairwise estimates (Hill, 1997).

As previously mentioned, change scores were calculated to represent change in participants' accuracy at using the HMR system and estimating portion size and food intake. Change scores were calculated by subtracting the accuracy score at the end of the trial (week 22-24) from baseline (week $1-4)$. The accuracy scores reflect percentage accuracy, where 0 represents perfect accuracy, 80 represents overestimation by $80 \%$, etc. Analysis of covariance indicated that the groups did not differ significantly on change scores for HMR anchor points, $F(2,29) 0.79, P=0.46$, portion size, $F(2,29) \quad 0.41, P=0.67$, or food intake estimates, $F(2,29)$ $1 \cdot 16, P=0 \cdot 33$. Therefore, the $t$ tests to determine if the change scores differed significantly from zero did not consider group. The results of these tests indicated that participants had a significant improvement in their accuracy of using the HMR system, $t(32)-4.23, P<0.001$, and estimating portion size, $t(32)-2.19, \quad P<0.05$, and food intake, $t(32)-3.33$, $P<0.01$, when presented with food. Accuracy scores at baseline and the end of the trial are provided in Table 1 . The mean (SD) change scores for use of the HMR system, portion size and food intake were -55 (SD 74), -11 (SD 30) and -50 (SD 86), respectively.

\section{Change in accuracy, measured with the PACE, associated} with training

One of $44(2.3 \%)$ participants had missing data for weeks 21-24. The missing completely at random assumption was met, $\chi^{2}(15) 21 \cdot 5, P=0 \cdot 12$ (Little \& Rubin, 1987). Expectation maximisation was used to impute missing values.
Table 1. Mean and standard deviation (SD) accuracy scores for use of the HMR system and estimated portion size and food intake for baseline and the end of the trial

\begin{tabular}{|c|c|c|c|c|c|}
\hline & \multicolumn{2}{|c|}{$\begin{array}{c}\text { Weeks } \\
1-4 \\
\text { (baseline) }\end{array}$} & \multicolumn{2}{|c|}{$\begin{array}{l}\text { Weeks } \\
22-24\end{array}$} & \multirow[b]{2}{*}{$P$ value } \\
\hline & Mean & SD & Mean & $\mathrm{SD}$ & \\
\hline $\begin{array}{l}\text { HMR anchor point accuracy } \\
\text { score }(0=\text { perfect } \\
\text { estimation })\end{array}$ & 62 & 64 & 7 & 20 & $<0.001$ \\
\hline $\begin{array}{l}\text { Portion size accuracy score } \\
\quad(0=\text { perfect estimation })\end{array}$ & 30 & 28 & 19 & 16 & $<0.05$ \\
\hline $\begin{array}{l}\text { Food intake accuracy score } \\
(0=\text { perfect estimation })\end{array}$ & 78 & 60 & 28 & 45 & $<0.01$ \\
\hline
\end{tabular}

Accuracy scores were calculated from participants' responses when they were provided with food. Accuracy scores reflect percentage accuracy, where 0 represents perfect accuracy, 80 represents overestimation by $80 \%,-20$ represents underestimation by $20 \%$, etc. $P$ values (last column) indicate if the difference in the accuracy score at baseline (weeks 1-4) and weeks 22-24 differed significantly from zero based on $t$ tests.

The groups did not differ significantly on change scores for HMR anchor points, $F(3,39) 1 \cdot 34, P=0.28$, portion size, $F(3,39) 0.88, P=0.46$, or the energy content of foods represented in the PACE pictures, $F(3,39) 0 \cdot 61, P=0 \cdot 61$. Therefore, the $t$ tests to determine if the change scores differed significantly from zero did not consider group. These tests indicated that participants had a significant improvement in their accuracy of using the HMR system, $t(43)$ $-2.43, \quad P<0.05$, but the estimated portion size, $t(43)$ $-0.99, P=0.33$, and energy content of the foods represented in the PACE pictures, $t(43)-1.58, \quad P=0 \cdot 12$, did not improve significantly. The accuracy scores for baseline and the end of treatment are provided in Table 2. The mean (SD) change scores for use of the HMR system, portion size and energy content of foods represented in the PACE were -18 (SD 50), -4 (SD 24) and -13 (SD 56), respectively.

Table 2. Mean and standard deviation (SD) accuracy scores for use of the HMR system, estimated portion size and estimated energy content of foods represented in pictures on the PACE for baseline and the end of the trial

\begin{tabular}{|c|c|c|c|c|c|}
\hline & \multicolumn{2}{|c|}{$\begin{array}{c}\text { Weeks } \\
1-4 \\
\text { (baseline) }\end{array}$} & \multicolumn{2}{|c|}{$\begin{array}{l}\text { Weeks } \\
21-24\end{array}$} & \multirow[b]{2}{*}{$P$ value } \\
\hline & Mean & $S D$ & Mean & SD & \\
\hline $\begin{array}{l}\text { HMR anchor point accuracy } \\
\text { score }(0=\text { perfect } \\
\text { estimation })\end{array}$ & 17 & 43 & -1 & 15 & $<0.05$ \\
\hline $\begin{array}{c}\text { Portion size accuracy score } \\
(0=\text { perfect estimation })\end{array}$ & 23 & 24 & 19 & 16 & 0.33 \\
\hline $\begin{array}{l}\text { Food intake accuracy score } \\
(0=\text { perfect estimation })\end{array}$ & 29 & 45 & 16 & 26 & 0.12 \\
\hline
\end{tabular}

PACE, photographic assessment of calorie estimation.

Accuracy scores reflect percentage accuracy, where 0 represents perfect accuracy, 80 represents overestimation by $80 \%,-20$ represents underestimation by $20 \%$, etc. $P$ values (last column) indicate if the difference in the accuracy score at baseline (weeks 1-4) and weeks 21-24 differed significantly from zero based on $t$ tests. 


\section{Discussion}

This is one of the first studies to determine empirically if people can learn a calorie counting system and if estimated food intake improves with training. The results of this study demonstrate that people can be trained to use the HMR Calorie System $^{(}$, i.e. their use of the anchor points became more accurate. Additionally, training resulted in more accurate estimates of food intake. Training also resulted in more accurate estimates of the portion sizes of provided foods, although these improvements were not as robust. The results also suggest that PACE is a useful assessment tool to quantify participants' accuracy at using the HMR system and it demonstrated training effects, i.e. a significant improvement in use of the HMR system was found from baseline to month 6 . The PACE did not detect the training effects for estimated portion size or the energy content of the foods represented in pictures.

The degree to which the participants learned to use the HMR Calorie System ${ }^{\odot}$ and improved the accuracy of their estimates of food intake when presented with food was impressive. The data reported herein provide clear evidence that participants can learn the HMR Calorie System ${ }^{\odot}$ and learn how to estimate food intake more accurately, at least for the foods provided during this study. These results are encouraging, since there are few empirical data on the ability of people to learn calorie counting systems. The extent to which participants can accurately estimate food intake for other foods eaten in a free-living environment is not known from this study, but should be the focus of future research.

The finding that training significantly improved the accuracy of portion size estimates of provided food was promising and supports previous research (Howat et al. 1994), although the amount of improvement was modest. This finding is in line with previous studies that reported only modest improvements in portion size accuracy with training (Brown \& Oler, 2000). Based on the data from this study, it appears that portion size estimates are highly variable, which limits the ability to identify improvements in the accuracy of portion size estimates. Large variability has been reported in another study that used pictures to improve portion size estimates (Robson \& Livingstone, 2000). The modest improvements in the accuracy of portion size estimates and the more robust improvements in use of the HRM system suggest that improved accuracy of food intake estimates is largely the result of the participants' ability to determine the energy content of each unit (serving) of food more accurately.

The mean food intake accuracy score at baseline is presented in Table 1. This value is positive, indicating that participants overestimated food intake. This finding is inconsistent with much of the literature on self-reported food intake, which has found that people under-report food intake and that obese people tend to under-report to a greater degree than lean people (Schoeller et al. 1990). It is possible that our finding of over-reporting of energy intake is due to the following factors. First, much of the literature that has demonstrated that people underestimate food intake was based on weight loss treatment-seeking obese people who use penand-paper food records to record food intake. Some methods of self-report, however, have been found to overestimate food intake. For example, the United States Department of
Agriculture five-step multiple pass method was found to result in overestimates of food intake by nearly $10 \%$, particularly among normal and overweight women (Conway et al. 2003). The present study is one of the first studies that quantified energy intake estimates with the HMR system, and it is possible that this system results in more accurate estimates of food intake compared with pen-and-paper records, or the system might result in a bias of overestimation of food intake. Secondly, self-report methods have been found to result in accurate estimates of food intake among highly motivated, normal-weight, self-selected participants (Black et al. 1993). The present study was based on overweight individuals, many of whom enrolled in the study to improve their health through dietary and/or exercise intervention, and not all participants were seeking weight loss treatment. Additionally, these participants were highly motivated, as indicated by their ability to complete rigorous study demands with little attrition. Therefore, it is possible that this sample represented a highly motivated overweight sample, a hypothesis that is supported by additional data. Based on $7 \mathrm{~d}$ pen-and-paper food records that were collected over 5 weeks during the parent trial, participants in the CR, CR + EX, LCD and weight maintenance groups under-reported food intake by only $8 \mathrm{~kJ}$ ( $2 \mathrm{kcal}, 0 \%)$, $519 \mathrm{~kJ}(124 \mathrm{kcal}, 5.5 \%), 147 \mathrm{~kJ}(35 \mathrm{kcal}, 1.7 \%)$ and $1047 \mathrm{~kJ}$ (250 kcal, $8.7 \%)$, respectively.

Training effects did not differ by group, indicating that participants who received a calorie-restricted diet or a weight maintenance diet benefited equally from the training. These results suggest that the HMR system and PACE can be used in both weight loss and weight gain prevention programmes. Additionally, these methods would probably benefit people who are required to follow specific diets, such as diets low in sodium or saturated fat, due to health conditions or study demands. These tools could improve adherence to such regimens by helping people: (1) plan meals that consist of a specific macronutrient composition; and (2) estimate their energy and macronutrient intake and determine if it is consistent with the prescribed meal plan. In addition, the results of this study follow the suggestions of Livingstone \& Robson (2000) who note that methods are needed to identify effectively misreporters or people who require additional resources to learn a calorie counting system. Nevertheless, inaccurate estimates of energy and macronutrient intake will occur when food intake is not recorded; hence, efforts are needed to promote the recording of all foods that are eaten. Finally, this study provides a thorough empirical examination of a calorie counting system, which is consistent with the suggestion of Cypel et al. (1997) that more thorough reports of portion size measurement aids are needed in the literature.

This is the first study to use PACE. The PACE was a useful assessment tool, and it was easily used to quantify participants' responses. It also detected a significant training effect with the HMR Calorie System ${ }^{(}$. Nevertheless, the preliminary development of PACE is a significant weakness of the method. This study reports results from the first iteration of PACE, and the photographs were of modest quality. Refinement of PACE will probably improve its utility and ability to detect training effects. Nevertheless, the portability of PACE is a significant strength that makes it a useful tool to quantify the responses of participants who cannot travel to a research centre. In addition, PACE could be used to quantify learning 
of other calorie counting systems. Another study limitation is the lack of a control group that did not receive HMR system training. The present study used a within-subjects design, and each participant served as his/her own control. Hence, the results of the study support the conclusion that training resulted in significant improvements in the accuracy of participants' use of the HMR system and the accuracy of estimated portion size and food intake, but it cannot be concluded that the HMR system was solely responsible for this effect. Additional research is needed to determine if food intake estimates improve significantly after HMR training in comparison with a control group that is not exposed to the HMR system.

In conclusion, people can be trained to use the HMR Calorie System $^{(0}$ accurately. Additionally, people can be trained to estimate the portion size of provided foods and food intake more accurately. The PACE is a useful and portable assessment that can be used to quantify participants' responses. The PACE detected significant improvement in use of the HMR system, although the ability of PACE to detect training effects for estimated portion size and energy content of foods represented in the pictures remains unproven.

\section{Acknowledgements}

The authors thank the CALERIE participants and the remaining members of the Pennington CALERIE Research Team including: D. Enette Larson Meyer, Tuong Nguyen, Marlene M. Most, Anthony Alfonso, Catherine Champagne, Brenda Dahmer, Andy Deutsch, Paula Geiselman, Jennifer Howard, Jana Ihrig, Michael Lefevre, Darlene Marquis, Connie Murla, Jennifer Rood, Aimee Stewart and Vanessa Tarver. The authors also thank Jacqueline Stump, Leah Blaize and Lauren Ortego for their work and dedication, which made this study possible. Our thanks also go to Health and Nutrition Technology, Carmel, CA for providing us with the HealthOne formula used in the study, and to Health Management Resources $\left(\mathrm{HMR}^{\mathrm{TM}}\right.$; Boston, MA) for permission to use the HMR Calorie System ${ }^{\odot}$. Preliminary results from this study were presented at the Annual Meeting of the North American Association for the Study of Obesity, October 2005, Vancouver, British Columbia, Canada. This work was supported by the following grants: U01 AG20478 (PI: E.R.) and 1 K23 DK068052-01A2 (PI: C.M.). L.M.. is supported by a Training Fellowship awarded by the NHMRC of Australia (ID 349553).

\section{References}

Anderson JW, Brinkman VL \& Hamilton CC (1992) Weight loss and 2-y follow-up for 80 morbidly obese patients treated with intensive very-low-calorie diet and an education program. Am J Clin Nutr 56, 244S-246S.
Black AE, Prentice AM, Goldberg GR, Jebb SA, Bingham SA, Livingstone MB \& Coward WA (1993) Measurements of total energy expenditure provide insights into the validity of dietary measurements of energy intake. J Am Diet Assoc 93, 572-579.

Brown LB \& Oler CH (2000) A food display assignment and handling food models improves accuracy of college students' estimates of food portions. J Am Diet Assoc 100, 1063-1065.

Conway JM, Ingwersen LA, Vinyard BT \& Moshfegh AJ (2003) Effectiveness of the US Department of Agriculture 5-step multiple-pass method in assessing food intake in obese and nonobese women. Am J Clin Nutr 77, 1171-1178.

Cypel YS, Guenther PM \& Petot GJ (1997) Validity of portion-size measurement aids: a review. J Am Diet Assoc 97, 289-292.

Flegal KM (2005) Epidemiologic aspects of overweight and obesity in the United States. Physiol Behav 86, 599-602.

Heilbronn LK, de Jonge L, Frisard MI, et al. (2006) Effect of 6-month calorie restriction on biomarkers of longevity, metabolic adaptation, and oxidative stress in overweight individuals: a randomized controlled trial. J Am Med Assoc 295, 1539-1548.

Hill JO (2001) Monitoring tools in the new millennium: technology solutions for weight management, Chairman's introductory remarks. Obes Res 9, Suppl 5, 359S-360S.

Hill M (1997) SPSS Missing Value Analysis 7.5. Chicago, IL: SPSS Inc.

Howat PM, Mohan R, Champagne C, Monlezun C, Wozniak P \& Bray GA (1994) Validity and reliability of reported dietary intake data. J Am Diet Assoc 94, 169-173.

Little RJA \& Rubin DB (1987) Statistical Analysis with Missing Data. New York: Wiley.

Livingstone MB \& Robson PJ (2000) Measurement of dietary intake in children. Proc Nutr Soc 59, 279-293.

Moore's Extended Nutrient (MENu) Database [Computerized Database] (2000) Baton Rouge, LA: Pennington Biomedical Research Foundation.

Must A, Spadano J, Coakley EH, Field AE, Colditz G \& Dietz WH (1999) The disease burden associated with overweight and obesity. $J$ Am Med Assoc 282, 1523-1529.

Perri MG \& Corsica JA (2002) Improving the maintenance of weight lost in behavioral treatment of obesity. In Handbook of Obesity Treatment, pp. 357-379 [TA Wadden and AJ Stunkard, editors]. New York: Guilford Press.

Rapp SR, Dubbert PM, Buttross Y \& Burkett PA (1998) Reducing diabetics' food portion size errors by using feedback training. J Compliance Health Care 3, 115-124.

Robson PJ \& Livingstone M (2000) An evaluation of food photographs as a tool for quantifying food and nutrient intakes. Public Health Nutr 3, 183-192.

Schoeller DA, Bandini LG \& Dietz WH (1990) Inaccuracies in selfreported intake identified by comparison with the doubly labeled water method. Can J Physiol Pharmacol 68, 941-949.

USDA (2000) United States Department of Agriculture, Agricultural Research Service, Continuing Survey of Food Intakes by Individuals, 1994-1996, 1998.

Williamson DA, Allen HR, Martin PD, Alfonso AJ, Gerald B \& Hunt A (2003) Comparison of digital photography to weighed and visual estimation of portion sizes. J Am Diet Assoc 103, 1139-1145.

Williamson DA, Allen HR, Martin PD, Alfonso A, Gerald B \& Hunt A (2004) Digital photography: a new method for estimating food intake in cafeteria settings. Eat Weight Disord 9, 24-28. 